\title{
The Constitution of Weyl's Pure Infinitesimal World Geometry
}

\author{
C. D. McCoy*
}

8 July 2021

\begin{abstract}
Hermann Weyl was one of the most important figures involved in the early elaboration of the general theory of relativity and its fundamentally geometrical spacetime picture of the world. Weyl's development of "pure infinitesimal geometry" out of relativity theory was the basis of his remarkable attempt at unifying gravitation and electromagnetism. Many interpreters have focused primarily on Weyl's philosophical influences, especially the influence of Husserl's transcendental phenomenology, as the motivation for these efforts. In this article, I argue both that these efforts are most naturally understood as an outgrowth of the distinctive mathematical-physical tradition in Göttingen and also that phenomenology has little to no constructive role to play in them.
\end{abstract}

\section{Introduction}

From the contemporary point of view, the science of cosmology is founded on relativity theory, and relativity theory is founded on spacetime geometry. This conception of cosmology began to be set a little over 100 years ago when, not even a year after the completion of his substantial work on the general theory of relativity (in his 1916 paper "The Foundations of the General Theory of Relativity"), Einstein founded modern physical cosmology with his 1917 paper "Cosmological Considerations in the General Theory of Relativity." In that famous paper, Einstein turned the general theory of relativity towards the description of the physical universe at large, yielding the first relativistic cosmological model, the Einstein static universe. With those epoch-making steps taken, the geometrical foundation of general relativity and cosmology was located among the variably-curved geometries first conceived by Riemann in the mid-19th century.

Before the 19th century, the spatial geometry of the world was generally understood to be fundamentally and necessarily Euclidean. When awareness of the consistency of (homogeneous) non-Euclidean geometries eventually spread enough to undermine Euclidean geometry's hegemony in the latter half of the 19th century, a multitude of philosophical responses sprang up to make sense of its overthrow, ranging from conventionalism to various neo-Kantian attempts to rescue Kantian a priorism about geometry. Yet earlier, in the midst of the century, Riemann's revolutionary initial development of differential geometry had already opened the

*Underwood International College, Yonsei University, Seoul, Republic of Korea. email: casey.mccoy@yonsei.ac.kr 
way to describing - in a single mathematical framework-not only the constant-curvature nonEuclidean geometries but also ones whose curvature varied from place to place. While Riemann's vision of the intimate intertwining of physics and geometry (Laugwitz, 1999, Ch. 3) did not immediately lead to the application of this generalized geometry in physics (nor indeed did it directly influence Einstein), his manifolds and the geometrical objects that live with them nevertheless emerged as the essential ground for Einstein's development of the general theory of relativity.

In 1917, Weyl, the principal figure of interest in this paper, turned his attention to the mathematical foundations of the general theory of relativity and its underlying geometry, lecturing on the theory at the Eidgenössische Technische Hochschule (ETH) in Zürich (from which Einstein had not long ago departed). These lectures came to form the basis of his classic Raum-ZeitMaterie (Space-Time-Matter), first published in 1918 (reaching a fifth edition already by 1923 and a first English translation in 1922). At the same time, Weyl initiated a novel project with the aim of improving on the Riemannian geometrical foundation of general relativity (Weyl, 1918b), one which subsequently led to the remarkable unification (or so thought Weyl) of gravitation with electromagnetism (Weyl, 1918a, 1919b). As Weyl relates in Raum-Zeit-Materie, he was motivated by what he saw as an inconsistency in Riemann's program of "infinitesimal geometry," a last vestige of the "distant geometry" (Ferngeometrie) of Euclid which had, however, been overlooked by Riemann, namely, the admissibility of making length comparisons at spatially distant points. Generalizing Levi-Civita's work on parallel transport, Weyl introduced a new geometrical "connection" which subsumes Levi-Civita's idea and has the consequence of making both angle and length comparisons path dependent. This completed "near geometry" (Nahegeometrie) was called by Weyl pure infinitesimal geometry.

Weyl's motivations for developing pure infinitesimal geometry and his unified field theory have attracted the attention of many historians, philosophers, and physicists, in particular due to Weyl's reinterpretation of the core idea of pure infinitesimal geometry ("gauge" symmetry) into quantum physics. According to some interpreters, these developments have a surprising source in Husserl's philosophy of transcendental phenomenology, which they claim supplies the epistemological basis for Weyl's dissatisfaction with Riemannian geometry $\left.\right|^{1}$ Yet, while Husserl's philosophical influence on Weyl is unquestionable (indeed, it is explicitly noted by Weyl in Raum-Zeit-Materie as well as his other important work from this period, Das Kontinuum), it seems to me that such reductive accounts of the origin of pure infinitesimal geometry in phenomenology only achieve their "streamlined finality" (Ryckman, 2005, 80) at the rather considerable cost of a good bit of inattention to historical and philosophical detail.

My aim in this paper is to correct the inattention to show that such interpretations of Weyl's work are in error. As for overlooked historical detail, the evidence (particularly that adduced from Scholz's numerous papers on Weyl and geometry, e.g. (Scholz, 1994), and from Weyl's own recollections) makes it clear that Weyl's primary intellectual influences in the development of pure infinitesimal geometry are those issuing from the Göttingen mathematical-physics tradition of which he is part-and ultimately and above all, Riemann, the decisive figure in this tradition. I make an economical, but in my view decisive, case for this point in section two. Then, as for overlooked philosophical detail, I point out that Weyl could hardly have been a faithful disciple of Husserlian philosophy in devising pure infinitesimal geometry, for the simple reason that his "philosophical" justification of pure infinitesimal geometry is substantially at odds with basic principles of phenomenology 2 I make the case for this point in section three,

\footnotetext{
${ }^{1}$ In this paper, I focus primarily on Ryckman's contributions, whose book Reign of Relativity (Ryckman, 2005) in particular is the most significant contribution espousing this perspective.

${ }^{2}$ I note that similar points to these two are mentioned in passing in (Eckes, 2014, 130-1) —although they are
} 
a point which I am not aware has been elaborated in the literature so far. These two points together demonstrate that the real, decisive motivations driving Weyl towards pure infinitesimal geometry are basically scientific in character, not philosophical. Establishing this is the specific aim of this paper.

If a philosophical influence must be identified for Weyl at this stage of his life, then, as Scholz (1995) and Sieroka (2007, 2009) have convincingly shown, his philosophical thinking is actually much more in line with the transcendental idealism of Fichte (as communicated to him especially by his colleague at the ETH, Fritz Medicus) rather than Husserl. The general aim of this paper, however, is not to isolate the true philosophical stratum of Weyl's ideas but instead to widen the lens of our historical appreciation of Weyl's distinctive style of thought, a style of thought that acts and is conditioned in a broad context (mathematical, physical, cultural, etc.). In my view, the historical appreciation of Weyl's distinctive style of thought has, however, been deleteriously foreshortened by excessive attention (by philosophical interpreters especially) on Weyl's wide-ranging philosophical interests. For this reason, I close the paper with some remarks on historical interpretation, recalling especially the view of R. G. Collingwood on the subject, and what Weyl himself had to say on his own place with respect to philosophy and mathematics.

\section{Weyl and Geometry}

In order to appreciate Weyl's thinking about geometry and its epistemology, it is necessary to first describe the key mathematical differences between Euclidean, Riemannian, and Weyl geometries. Due to limitations in space, however, I will have to avoid all mathematical complexities here ${ }^{3}$ Following this brief geometrical introduction, I will then turn to certain received interpretations of Weyl's justification for pure infinitesimal geometry by way of Husserlian phenomenology.

For the sake of simplicity in illustrating the basic geometrical issues, let us first fix attention on a two-dimensional space. If this space has a Euclidean geometry, then certain comparisons evidently make sense "at a distance." For example, one can compare both the length and direction of an arrow whose base is attached to one point to those of an arrow located at any other point. We can, as it were, detach one arrow in our mind's eye and move it, without making any other modification, to the other and make the comparison directly (using the Euclidean "metric" to make the comparison). In short, in Euclidean geometry there is simply no need to consider "how" one effects the transportation of an arrow from one point to another in order to make a comparison; any manner will do, so long as we do not rotate or distort it.

Riemannian geometry generalizes Euclidean space in a way that makes it generally necessary to consider how to effect such a comparison however. Consider the (intrinsic) twodimensional geometry of the surface of a sphere (i.e., without reference to an ambient space in which it might be imbedded). How does one know how an arrow attached to a point at the equator compares to an arrow attached to one of the poles? Different ways of moving one arrow along the surface of the sphere to the other to make the comparison will yield different results. Levi-Civita's great contribution to geometry was in establishing a standard for what it means geometrically to "move an arrow along a curve"- that is, what is called parallel transport. Parallel transport of an arrow involves (roughly speaking) maintaining the relative angle between

not explicitly argued for or elaborated on in the paper.

${ }^{3}$ Detailed expositions of the mathematics involved can be found in (Scholz, 2001), (Coleman and Korté, 2001, §), and (Ryckman, 2005, Ch. 6). 
the arrow and the tangent line of the curve being followed. Take an arrow pointing along the sphere's equator and transport it along the equator for a distance of one quarter of the sphere's circumference, maintaining its orientation along the way; then transport it along a line of longitude directly to the north pole, maintaining its orientation along this line as well. Now take the same original arrow and transport it directly to the pole. One should find that the resulting two arrows will be at right angles to one another. This simple example clearly demonstrates how comparison of angles in Riemannian geometry is (in general) path-dependent. A moment's reflection will indicate that this novel feature of Riemannian geometry is readily explained by the space itself possessing an intrinsic curvature (which, it turns out, is entirely captured by the Riemannian "metric" of the curved space).

Note, however, that the length of the arrow intuitively remains unchanged by transport along a path. Therefore, while relative orientations can only be compared by parallel transporting one arrow to the other, length comparisons can still be made "at a distance" in Riemannian geometry, in the same sense that both relative orientations and magnitudes can be compared "at a distance" in Euclidean geometry. Weyl's pure infinitesimal geometry is a generalization of Riemannian geometry which allows that the transport of an arrow along a curve might result in changes to its length as well, depending on the path it follows through the geometry. In this case, the analogous "parallel transport" of the arrow involves maintaining both the relative magnitude and the relative angle of the arrow with fiducial arrows along the curve. These arrows are "tangent vectors" determined by the "infinitesimal" behavior of the curve in the neighborhood of each point. It follows from this that in pure infinitesimal geometry it is not possible to directly compare angles or magnitudes of arrows at a distance, for both comparisons (at least in general) become path-dependent ${ }^{4}$ Moreover, it becomes possible to transport an arrow from one point along a path back to its starting point only to find that its length has changed. This feature makes pure infinitesimal geometry a complete "near geometry," unlike Riemannian geometry, which is a "near geometry" only with respect to angles.

Thinking about the geometry of surfaces clearly suggests the good sense of Riemann's generalization of Euclidean geometry. But what justifies Weyl's further generalization (particularly given the apparent lack of an intuitive example of such geometry)? According to Ryckman's prominent interpretation, pure infinitesimal geometry is actually the result of an application of the epistemological framework of Husserlian phenomenology. Not entirely, to be sure, for "while Husserlian phenomenology indeed sets the epistemological framework of Weyl's reconstruction, its particular steps, mathematical constructions, and especially its 'style' manifest very much Weyl's own creative endeavor to interpret and implement the method of phenomenological investigation in the context of a fundamental physical theory" (Ryckman, 2005, 146). Nevertheless, the role of phenomenological thinking is accorded primacy by Ryckman (as well as others who have followed him) ${ }^{5}$

According to Ryckman, the "phenomenological" way to pure infinitesimal geometry begins from the fundamentally idealistic thesis that the world exists only for consciousness. As Weyl himself states this credo in the introduction to Raum-Zeit-Materie, "the real world, each of its constituent parts and all their determinations, are and can only be given as intentional objects of acts of consciousness" (Weyl, 1919a, 3, my translation). Such a formulation, particularly

\footnotetext{
${ }^{4}$ And thus, analogously to the Riemannian case, there is a novel "length curvature" in the spaces of pure infinitesimal geometry.

${ }^{5}$ E.g.: "Hermann Weyl implements Husserlian phenomenology in the foundations of general relativity by means of a sophisticated constructive mathematical development of what he calls purely infinitesimal geometry" (Friedman, 2013, 97); "Weyl's 'purely infinitesimal' understanding of nature is an epistemological concomitant to the constitutive transcendental-phenomenological idealism of Husserl” (Ryckman, 2005, 82).
} 
with its emphasis on intentionality, indeed bears the unmistakable mark of Husserl's distinctive manner of writing. According to Husserl, while we normally regard the world as existing in its own right, independently of any conscious entity, reflection on our conscious experiencing itself (specifically through what is called the "phenomenological reduction") leads to the recognition that the transcendent world of things is not absolute but relative, dependent on subjectivity for the possibility of its constitution; reality is real, but only in the sense that it can be given intuitively as an objective correlate of consciousness.

Weyl, however, adds a novel proviso to his general agreement with (Husserlian) idealism, claiming that the ego can only comprehend the material world with what is "immediately" available to it in experience. As he puts it, "only to spatio-temporal coincidence and immediate spatio-temporal proximity can we assign an intuitively evident meaning" (Weyl, 1949, 95). By immediate spatial-temporal neighborhood, Weyl specifically intends what is "infinitesimally" nearby (in contemporary terms, what is localizable at a point or in that point's tangent space). From Weyl's epistemological point of view, then, Riemannian (and Euclidean) geometry are inadequate, because they (ostensibly) presuppose geometrical facts that are not "infinitesimal," such as making direct comparisons of magnitude "at a distance." In short, as certain facts are presupposed (a priori, as it were) that are inaccessible to intuition (given in experience), they are epistemologically inadmissible. Weyl elaborates his view as follows:

The Riemannian geometry referred to above still contains a residual element of [distant] geometry [Ferngeometrie]—without any substantial reason, as far as I can see. It seems to be due to the accidental origin of this geometry in the theory of surfaces. The quadratic form [i.e., metric] enables us to compare, with respect to their length, not only two vectors at the same point, but also the vectors at any two points. But a truly infinitesimal geometry [Nahegeometrie] must recognize only the principle of the transference of a length from one point to another point infinitely near to the first. This forbids us to assume that the problem of the transference of length from one point to another at a finite distance is integrable [i.e., pathindependent], more particularly as the problem of the transference of direction has proved to be non-integrable [i.e., path-dependent]. (Weyl, 1923, 203)

Nevertheless, Weyl does accept that some geometrical structure must be presumed a priori, namely the topological structure of space(time):

We now distinguish between the amorphous continuum and its metrical structure. The first has retained its a priori character, but has become the counterpart [Gegenbild] of the pure consciousness standing freely opposed to Being, while the structural field [i.e., metric field] is completely surrendered to the real world and its play of forces. (Weyl, 1931, 51, my translation)

This crucial conceptual separation of the "a posteriori" metrical structure of a space from its "a priori" topological structure is no invention of Weyl's (and certainly not Husserl's) but rather traces back to Riemann. As Weyl explains,

Riemann had an entirely different conception of the nature and origin of the metrical properties of space. For him the metric field is not given rigidly once and for all, but is causally connected with matter and thus changes with the latter. $\mathrm{He}$ considers the metric not as part of the static homogeneous form of phenomena, but of their ever-changing material content. (Weyl, 1949, 86-7) 
Weyl further relates the Riemannian connection by explaining that Riemann was fundamentally motivated by the aim to understand the physical world from its behavior in the infinitely small, ultimately connecting Riemann's idea to Leibniz's continuum conception of physics:

As the true lawfulness of nature, according to Leibniz's continuity principle, finds its expression in laws of nearby action, connecting only the values of physical quantities at space-time points in the immediate vicinity of one another, so the basic relations of geometry should concern only infinitely closely adjacent points ("near-geometry" as opposed to "far-geometry"). Only in the infinitely small may we expect to encounter the elementary and uniform laws, hence the world must be comprehended through its behavior in the infinitely small. (Weyl, 1949, 86)

Riemann's motivations for going beyond Euclidean geometry do not emerge from the influence of any idealistic philosophy however. Rather, his view on method was typically scientific in character:

The decision of these questions [i.e., on the validity of the presuppositions of geometry in the infinitely small] can only be found by proceeding from the conception of phenomena previously proven by experience (for which Newton laid the foundation) and gradually reworking it, driven by facts which it cannot explain. Such investigations, which, like the one carried out here, proceed from general concepts, can only serve to insure that this work is not hindered by the limitation of concepts, and that progress in understanding the innermost connection of things [Zusammenhangs der Dinge] is not inhibited by traditional prejudices. (Riemann, 1919, 20-1, my translation)

This matter of avoiding conceptual hindrance in mathematics took on a special significance for Riemann, for he believed in a "preestablished harmony" between mathematics and physics; indeed, according to Pyenson, "preestablished harmony was the engine that drove his grandiose project forward" (Pyenson, 1982, 153). Riemann's fundamental influence on the emerging Göttingen tradition of mathematics saw many of his ideas adopted by Klein and Hilbert, both of whom "were exponents of the Leibnizian notion that a 'preestablished harmony' exists between mathematical ideas and physical reality" (Rowe, 1989, 212), and which were transmitted by them to their student Weyl. ${ }^{6}$

Weyl's pure infinitesimal geometry is thus most easily understood as a natural technical development of the mathematical-physical geometrical field physics of Riemann and the Göttingen tradition that follows from him. Echoing Riemann's statement above, pure infinitesimal geometry is a modification of the "proven by experience" Riemannian geometry of general relativity, the product of an investigation which aims to insure that physics is not hindered by overly restricted concepts, and is motivated by the idea of understanding the world's behavior in the infinitely small. Although my discussion here is intended to make this claim sufficiently plausible to the reader, it is further grounded in extensive historical work, especially the many studies on Weyl by Scholz.7 It is also evident from Weyl's own recollections (rather than his philosophical rhetoric in, e.g., Raum-Zeit-Materie). For example, in (Weyl, 2009a, 168), he recalls the initial impetus to his development of pure infinitesimal geometry as a conversation

\footnotetext{
${ }^{6}$ Minkowski too promoted this idea of a pre-established harmony at Göttingen and promulgated it to Weyl. See (Eckes, 2014).

${ }^{7}$ Scholz explicitly makes my point in (at least) one place, saying "Weyl's infinitesimal geometry of the years 1917 to 1925 builds on Riemann's motives in many ways and, in particular, quite consciously on the Riemannian tradition cultivated in Göttingen" (Scholz, 2001, 97, my translation).
} 
with a student, which triggered the realization (helped by his Göttingen tradition-inspired attraction to Mie theory) that making length path-dependent in geometry opens up the possibility of a unification of all known field physics (i.e., electromagnetism with gravitation).

Despite all this, might one still try to read Weyl's pure infinitesimal geometry as an "implementation" of Husserlian phenomenology? A concomitant to Husserlian transcendental idealism? Perhaps-but I believe it is only possible in some minimal proportion. It is surely not my aim to suggest that Husserlian ideas are totally absent in Raum-Zeit-Materie or irrelevant to its author's thinking. But in judging this proportion, one might first bring up the fact that Husserl's name appears only once in the main text of Raum-Zeit-Materie, in comparison to Gauss (13 times) and Riemann (14 times). Moreover, the oft-cited passages in the book which evoke distinctively Husserlian language (which, of course, are accorded great significance by philosophical interpreters), in fact comprise little more than a couple of pages of the entire book (Weyl, 1919a, 3-4)-in the midst of philosophical remarks that could equally well be read as inspired by Kant or Fichte ${ }^{8}$ Of course, Weyl need not name Husserl or recapitulate his philosophy in order to carry out a phenomenology-motivated project. However, as I will now turn to arguing, I do not think we can regard Weyl's project of pure infinitesimal geometry as significantly motivated or justified by the actual tenets of Husserlian phenomenology.

\section{Weyl and Phenomenology}

As said, numerous commentators have situated Weyl's thought in the context of Husserlian phenomenology, and, while good cases can be (and have been) made about Husserl's influence on Weyl, especially concerning the continuum and towards transcendental idealism, I believe it would be a gross mistake to portray Weyl as primarily working within a specifically phenomenological paradigm-let alone as a "disciple of Husserl"-particularly when it comes to his work in geometry and physics. Nevertheless, such accounts of Weyl's thought on these topics have become influential, especially as developed in the chapters on Weyl in Ryckman's book The Reign of Relativity. Ryckman's project there is to "identify, and form a coherent picture of, the various currents of Husserlian transcendental-phenomenological idealism within Weyl's writings" (Ryckman, 2005, 110); on the basis of this analysis, Ryckman concludes that Husserlian "themes are seen to be salient in [Weyl's] 'broadening' of relativity theory...in particular...how 'transcendental subjectivity,' in Husserl's sense of the 'absolute being' of 'pure consciousness' surviving the phenomenological reduction, plays the fundamental role in Weyl's understanding of the constitution of objectivity in physical theory" (Ryckman, 2005, 110). The previous section was intended to make the case that conclusions like these "put the cart before the horse," by putting Weyl's philosophical motivations ahead of his scientific ones; the present section will reveal the contents of that cart to also be a "sallow bill of goods" (Ryckman, 2005, viii). I make two key points: 1) Weyl is not carrying out a properly phenomenological investigation in any substantive sense, and 2) Weyl's "epistemological" assumptions are not actually licensed by phenomenology.

Let me begin with a brief elaboration of Husserlian phenomenology. In its mature form, it is a kind of transcendental philosophy, whose principal aim is to carry out a presuppositionless investigation of the structures of consciousness. Husserl investigated various pathways into the subject matter of phenomenology over his career (e.g., the Cartesian way, the ontological way,

\footnotetext{
${ }^{8}$ Even the telltale appearance of Husserlian philosophy in the introduction can be easily explained away, for "at the time more esteem could be expected among mathematicians, in particular from the Göttingen milieu, from references to Husserl's philosophy" (Scholz, 2005, 338).
} 
etc.), but all involve making a decisive break with a certain pervasive metaphysical attitude toward the world and worldly objects. Husserl calls this attitude the "natural attitude" (Husserl, 2014[1913]). In the natural attitude, we uncritically regard the world and all the objects within it as simply existent (e.g., as mind-independent). Although there is nothing about this attitude to object to when carrying out the practical activities of our daily lives, philosophically speaking it is a dogmatic presupposition that stands in the way of a truly critical inquiry into the nature of the world and the proper justification for our beliefs about it. To carry out such an inquiry, it is essential to "bracket" the natural attitude in a move which Husserl calls the epoché. We do not thereby engage in radical doubt of the world or our beliefs about it (Husserl, 1999[1931]); rather, we simply withhold this attitude's validity for us in order to clear the way to adopt what Husserl calls the "phenomenological attitude." In the phenomenological attitude, we are understood to be free to reflect thematically, without presupposition, on the world and its correlation with consciousness in conscious experience.

The phenomenologist's principal aim, according to Husserl, is to study the givenness of reality in experience. One of Husserl's central insights is that a cardinal feature of our conscious experience is that it is intentional in character (an idea refashioned from Husserl's teacher Brentano). That is to say, it is directed, it is about things, things which are themselves given to us in our conscious experiences. Perception provides a simple illustration. When I perceive a lamp on the table, I obviously only perceive the lamp itself partially (the entire lamp necessarily "transcends" my perception of it-it is in no way "contained" in my experience). Nevertheless, reflection immediately reveals to me that my experience is not of a "lamp-perspective" but indeed of the lamp itself. The latter is, as Husserl says, the intentional object of my lampexperience, which is directed towards it - and not merely the partial perspective of it, the sensations I have of it, or some independently existing object that happens to be appropriately "causally" related to me.

Certainly, we can see many of these basic phenomenological ideas indicated in the introduction to Weyl's Raum-Zeit-Materie in Husserl's distinctive idiom:

In full generality, the real [wirkliche] world, each of its constituent parts and all their determinations, are and can only be given as intentional objects of acts of consciousness. The absolute given are the conscious experiences which I havejust as I have them. Now of course, they by no means consist, as the positivists often assert, of some mere stuff of sensation, but in a perception: for example, for me there is in fact an object bodily there, to which that experience is related, in a completely peculiar way known to everyone but which cannot be further described, which according to Brentano is to be denoted by the expression "intentional object." When I perceive, I see this chair, for example, I am fully directed towards it. I "have" the perception, but only when I make this perception anew into the intentional object of a new, inner perception, which I am capable of in a free act of reflection, do I "know" something about it (and not just about the chair), and ascertain what I said just above. In this second act the intentional object is an immanent one, namely, like the act itself, a genuine [reelles] component of my stream of experiences; in the primary act of perception, however, the object is transcendent, i.e. given in an conscious experience but not a genuine [reelles] component of it. (Weyl, 1919a, 3-4, my translation)

A prominent feature of Husserl's philosophy, of particular significance to Weyl, is the "intuition of essences" (Wesensschau), which Husserl also calls "eidetic intuition" (the intuition of essential forms). To be sure, Husserl's view that the scope of intuition went beyond Kant's 
merely sensuous intuition (i.e., present-ness in perception) was attractive to Weyl. Although the 19th century saw a decisive move in German mathematics away from the primacy of sensuous intuition towards a more "conceptual style" (Ferreirós, 2007, Ch. 1), philosophically grounded instead in the Kantian "understanding," Weyl was nevertheless drawn to the belief that real mathematical knowledge is fundamentally grounded in intuition ("immediate insight"). In Das Kontinuum, for example, Weyl criticizes Dedekind's famed motto, that "nothing capable of proof ought to be accepted in science without proof":

This remark is certainly characteristic of the way most mathematicians think. Nevertheless, it is a preposterous principle. As if such an indirect concatenation of grounds, call it a proof though we may, can awaken any "belief" apart from assuring ourselves through immediate insight that each individual step is correct. In all cases, this process of confirmation - and not the proof-remains the ultimate source from which knowledge derives its authority; it is the "experience of truth." (Weyl, 1994, 119, n. 19, my emphasis)

According to Ryckman, it is precisely "Husserl's phenomenological method of eidetic analysis [that] guided Weyl in arriving at, and in mathematically formulating, his "pure infinitesimal geometry' (Ryckman, 2005, 111). Phenomenology, being focused on the analysis of essential structures of consciousness and limited to only what can be intuited from conscious experience, promises essential insight into consciousness, insight which Husserl holds can only be founded on eidetic intuition. Husserl's suggested method for obtaining it is called eidetic variation or the eidetic reduction. It involves the variation in imagination of something originally given in conscious experience in order to discern what is essential to it. Eidetic variation is not, however, a distinctively phenomenological process (contra Ryckman), for it is one that we can perform equally well within the natural attitude, mathematics being the most salient example where such a process may be employed to advantage (Tieszen, 2005; Lohmar, 2010). Besides that, as Zahavi points out, "interest in essential structures is so widespread and common in the history of philosophy that it is nonsensical to take it as a defining feature of phenomenology" (Zahavi, 2003, 37). Thus, although Weyl may well have been in part guided by the idea of eidetic analysis in formulating pure infinitesimal geometry, his acknowledgment of the epistemological significance of intuitive insight cannot, by itself, suffice to characterize any of his work in physics or mathematics as substantially phenomenological or as "implementing" phenomenology.9

There is even less reason to think that Weyl's approach to mathematics and physics is phenomenological in some other way. As da Silva says, "if Weyl were content with simply describing what he found in his intuitions, as an orthodox phenomenologist would probably be happy to do...he would not be much concerned with trying to 'reconstruct' it in symbolic terms and reshaping it in terms of mathematical concepts. (da Silva, 1997, 279). Physics and mathematics, like all typical products of human activity, are activities carried out in the natural attitude. Indeed, the characteristic objectivizing of the natural world in physics, in its distinctively symbolic and third-personal way, is a sublimated form thereof. Phenomenological investigations, by their very nature, cannot disclose facts and forms of the geometry or physics of the natural world. That, naturally enough, is rather the task of mathematicians and physicists. (Nonetheless, with regard to their task, phenomenology does indeed have a task properly of its own, namely, the task of uncovering the transcendental grounds of such scientific activity.)

\footnotetext{
${ }^{9}$ Moreover, according to da Silva's analysis, "although arguably Weyl's intuition-based epistemology was inspired by Husserl (as we can infer from the preface of [Das Kontinuum]), he seems to derive his conception of intuition from Poincaré and Brouwer rather than Husserl himself" (da Silva, 2017, 331).
} 
My opponents might concede this much, that Weyl is not really pursuing a phenomenological investigation in his mathematical work, and propose instead that his project merely respects the epistemological deliverances of Husserlian phenomenology. Certainly, Husserl demands that physics and mathematics not be empty symbolic games; they must have sense-in his later terms, by being ultimately founded in the "lifeworld," the pre-scientific world of practically oriented experience. Weyl's "phenomenological requirements on evidence" (Ryckman, 2020, 165), however, mandate something rather different than this. As Weyl says,

Only the spatio-temporally coinciding and the immediate spatial-temporal neighborhood has a directly clear meaning exhibited in intuition.... The philosophers may have been correct that our space of intuition bears a Euclidean structure, regardless of what physical experience says. I only insist, though, that to this space of intuition belongs the ego-center and that...the relations of the space of intuition to that of physics, becomes vaguer the further the distance from the ego-center. (Weyl, 1931, 49, 52)

This "théorème épistémologique" (Lobo, 2019, 38) of Weyl's, however, does not make any sense as a principle of phenomenology. When I reflect on my perception of objects that surround me, I immediately recognize these objects as being at various distances-I certainly perceive them as beyond my "immediate spatial-temporal neighborhood"-and they do indeed have a directly clear meaning to me precisely as such. On the basis of this rather basic phenomenological reflection alone, it already does not seem as if there could be a good phenomenological rationale for restricting the intuitions to be used for physical representation to my "infinitesimal neighborhood." To be sure, I am an embodied person, whom I experience as such and as being extended and located in space, there among the various objects that surround me. Yet it would be a fundamental misunderstanding of Husserl's view, one which Weyl unfortunately seems to be making, to think that the intuitions I have of surrounding objects somehow "take place" at my "ego-center," as if they were private mental items that are located where my body is.

How, then, could Weyl reason that some subjective "space of intuition" should be superimposed on an objective, physical representation of space? To answer this question, it is useful to elaborate somewhat on Husserl's views on space and geometry. According to the various manuscripts (never published during his lifetime) where Husserl discusses geometry, and as discussed by Mohanty (2008, ch. 2) and da Silva (2012), Husserl distinguishes between several genetically-related geometrical spaces which arise at different "levels of constitution": first, and fundamentally, there is the space of perception (space as we know it in everyday life from the arrangement of objects, as considered in the previous paragraph); second, the space of pure geometry (a mathematical representation of perceptual space obtained by abstraction and idealization of empirical shapes); third, the further abstracted spaces of pure mathematics (non-Euclidean space, Riemannian space, etc.), the spaces utilized in mathematical theories of physics (where methodological, scientific considerations play a significant role in determining their character); fourth, a possibly transcendentally real metaphysical space. While the first can be said to be at best "proto-Euclidean," the second is regarded by Husserl as (to a good approximation) Euclidean. The spaces of physics and mathematics, however, can in principle have any geometry whatever for Husserl, as they involve additional idealizations, abstractions, and modifications. For Husserl, then, even if it is correct to say that our ("ego-centered") space of intuition bears a Euclidean structure (he thinks it does), at most what that fact requires is that the space implemented in a model of physics be locally approximated by Euclidean space (so that it can be coherently related to the intuitive space of experience). That, however, is a 
relatively weak requirement, one which allows that physical space may even be described as discrete (as Riemann also allowed, incidentally), as it is conceivable that a Euclidean geometry may "emerge" out of a discrete underlying manifold.

Weyl's hypothesis - that "physics adheres to the Euclidean quality of the infinitely small neighborhood of a point $O$ (at which the ego happens to be at the moment)" (Weyl, 1949, 135, my emphasis) - in effect mandates a strong Euclidean requirement from the second level of constitution, the space of pure geometry, on infinitesimal neighborhoods in the third level of constitution, which spaces are to be imbedded in an abstract geometry of his devising, one which he independently takes to be the necessary form of physical space. In combining Euclidean spaces in this way, Weyl is driven by the principle that "the geometrical structure of physical space must reach a compromise between intuition and logical, methodological or theoretical requirements of science" (da Silva, 2012, 29). As we have just seen, Husserl would accept some such compromise as well, without, however, collapsing spaces that are intuitively distinct in their constitution. Weyl's particular solution thus can find no special satisfaction in phenomenological methods, for these levels of constitution must be taken according to their individual essences and by their genetic relations to one another. ${ }^{10}$

\section{Weyl Between Mathematics and Philosophy}

I will conclude by saying something of more general significance which has informed my composition. It is simply this. If we wish to understand Weyl and his individual development of pure infinitesimal geometry, which is to say, to understand these things historically, we must above all strive to understand his distinctive manner of thought, his particular frame of mind, in carrying out its development. In this vein, Collingwood had the following to say:

You are thinking historically...when you say about anything, "I see what the person who made this (wrote this, used this, designed this, \&c.) was thinking." Until you can say that, you may be trying to think historically, but you are not succeeding. (Collingwood, 2013, 110)

If some one, hereinafter called the mathematician, has written that twice two is four, and if some one else, hereinafter called the historian, wants to know what he was thinking when he made those marks on paper, the historian will never be able to answer this question unless he is mathematician enough to think exactly what the mathematician thought, and expressed by writing that twice to are four. (Collingwood, 2013, 111).

Echoing Collingwood, I believe we will fail to understand Weyl (i.e., we will not succeed in thinking historically about Weyl), if we strive to interpret him after some particular fashion, such as "phenomenologically" or "transcendentally idealistically," rather than simply trying to see things from his own point of view. It is clear when reading him (and looking through what he did) that Weyl thinks in the first place like a scientist. Indeed, we see him say it about his own work in Raum-Zeit-Materie:

I myself am also unable to give such answers to the attendant epistemological questions which I could fully accept in good epistemic conscience [Erkenntnisgewissen]. The ideas to be presented here did not result from some speculative

\footnotetext{
${ }^{10}$ I regret that space considerations do not allow me to extend the considerations raised in this section to Weyl's treatment of the problem of space.
} 
immersion into the foundations of physical knowledge, but rather developed from concrete physical problems encountered in the expansion of living, ever advancing [vorwärts drängenden] science, to whom the old shell had became too narrow. (Weyl, 1919a, 2).

That Weyl reminds us here that it is scientific problems which drive the practice of science forward brings to mind the following well-known remark, much in the same vein, by Einstein, another scientist much engaged by philosophy:

The scientist...accepts gratefully the epistemological conceptual analysis [of the philosopher]; but the external conditions, which are set for him by the facts of experience, do not permit him to let himself be too much restricted in the construction of his conceptual world by the adherence to an epistemological system. $\mathrm{He}$ therefore must appear to the systematic epistemologist as a type of unscrupulous opportunist. (Schilpp, 1949, 684)

Weyl was by no means a mere spectator of philosophy, of course, even if he encountered philosophical thinkers "like a butterfly who, as it flies from flower to flower, endeavors to get a bit of honey from each one" (Weyl, 2009b, 220). He also emphasizes the importance of philosophical reflection to science in the final words of the introduction of Raum-Zeit-Materie:

Especially the mathematician, who in his scientific training [ausgebildeten Wissenschaft] works with his concepts in strict and formal ways, needs to be reminded from time to time that the origins point back to darker depths than he is able to grasp with his methods. Beyond all knowledge of individual facts, there remains the task of comprehending. Despite the discouraging swaying back and forth of philosophy from system to system, we cannot do without it if knowledge is not to turn into senseless chaos. (Weyl, 1919a, 9, my translation)

Should we regard Weyl then as a "philosopher"? A "transcendental idealist"? If a label must be applied, then, yes, Weyl thought philosophically, was undoubtedly attracted to this kind of philosophy, whether it came from Kant, Fichte, or Husserl. Nevertheless, Weyl, in his own words, has this to say:

It seems to me that in the intellectual life of men two spheres can be distinguished, the one that of doing, shaping, constructing, creating something, in which the active artist, scientist, technician, statesman move, the other that of reflection where the meaning of all this activity is questioned and which one may consider the proper domain of the philosopher. (Weyl, 2012, 191)

Weyl, like Einstein, stands out to us as a scientist because of how he moved between both spheres in his thought; nevertheless, for both the first sphere was the primary sphere of their thought and activity. If the motivations of this sphere are not clear to us, then it seems to me that we, particularly the philosophers among us, will certainly fail to "think historically" about scientists, and the significance of what they have done, shaped, constructed, and created.

\section{References}

Coleman, Robert Alan, and Herbert Korté. 2001. "Hermann Weyl: Mathematician, Physicist, Philosopher." In Hermann Weyl's Raum - Zeit - Materie and a General Introduction to His Scientific Work, ed. Erhard Scholz, 157-386. Basel: Springer. 
Collingwood, R. G. 2013. "An Autobiography." In R. G. Collingwood: An Autobiography and Other Writings, ed. David Boucher and Theresa Smith, 1-172. Oxford: Oxford University Press.

da Silva, Jairo José. 1997. “Husserl's Phenomenology and Weyl's Predictivism." Synthese 110: 277-296.

2012. "Husserl on Geometry and Spatial Representation.” Axiomathes 22: 5-30.

- 2017. "Husserl and Weyl." In Essays on Husserl's Logic and Philosophy of Mathematics, ed. Stefania Centrone, 317-352. Dordrecht: Springer.

Eckes, Christophe. 2014. "Weyl et la geómétrisation de la physique." Revue d'Histoire des Mathématiques 20: 95-170.

Ferreirós, Jóse. 2007. Labyrinth of Thought, 2nd ed. Basel: Birkhäuser.

Friedman, Michael. 2013. "Transcendental Philosophy and Modern Physics: Neo-Kantianism, Logical Empiricism, and Phenomenology." In Reference, Rationality, and Phenomenology, ed. Michael Frauchiger, 89-106. Frankfurt: Ontos.

Husserl, Edmund. 1954/1970. The Crisis of European Sciences and Transcendental Phenomenology. Evanston, IL: Northwestern University Press.

_. 1931/1999. Cartesian Meditations. Dordrecht: Kluwer.

1913/2014. Ideas I. Indianapolis, IN: Hackett.

Laugwitz, Detlef. 1999. Bernhard Riemann, 1826-1866. New York: Springer.

Lobo, Carlos. 2019. "Le résidu philosophique du problème de l'espace chez Weyl et Husserl." In Weyl and the Problem of Space, ed. Julien Bernard and Carlos Lobo, 35-97. Cham: Springer.

Lohmar, Dieter. 2010. "Intuition in Mathematics: on the Function of Eidetic Variation in Mathematical Proofs." In Phenomenology and Mathematics, ed. Mirja Hartimo, 73-90. Dordrecht: Springer.

Mohanty, Jitendra Nath. 2008. The Philosophy of Edmund Husserl. New Haven, CT: Yale University Press.

Pyenson, Lewis. 1982. "Relativity in Late Wilhelmian Germany: The Appeal to a Preestablished Harmony between Mathematics and Physics." Archive for History of Exact Sciences 27: $137-155$.

Riemann, Bernhard. 1854/1919. Über die Hypothesen, welche der Geometrie zu Grunde liegen ed. Hermann Weyl. Berlin: Springer.

Rowe, David. 1989. "Klein, Hilbert, and the Göttingen Mathematical Tradition." Osiris 5: $186-213$.

Ryckman, Thomas. 2005. The Reign of Relativity. Oxford: Oxford University Press. 
2020. "Hermann Weyl, the Gauge Principle, and Symbolic Construction from the "Purely Infinitesimal"., In One Hundred Years of Gauge Theory, edited by Silvia De Bianchi and Claus Kiefer, 161-183. Cham: Springer.

Schilpp, Paul, ed. 1949. Albert Einstein: Philosopher-Scientist. Evanston, IL: Northwestern University Press.

Scholz, Erhard. 1994. "Hermann Weyl's Contribution to Geometry, 1917-1923." In The Intersection of History and Mathematics, ed. Sasaki Chihara, Sugiura Mitsuo, and Joseph Dauben, 203-230. Basel: Birkhäuser.

_. 1995. "Hermann Weyl's "Purely infinitesimal geometry"." In Proceedings of the International Congress of Mathematicians, ed. Srishti Chatterji, 1592-1603. Basel: Birkhäuser.

. 2001. "Weyls Infinitesimalgeometrie, 1917 - 1925.” In Hermann Weyl's Raum - Zeit - Materie and a General Introduction to His Scientific Work, ed. Erhard Scholz, 48-104. Basel: Springer.

2005. "Philosophy as a Cultural Resource and Medium of Reflection for Hermann Weyl." Revue de Synthèse 126: 331-351.

Sieroka, Norman. 2007. "Weyl's 'agens theory' of matter and the Zurich Fichte." Studies in History and Philosophy of Science Part A 38: 84-107.

2009. "Husserlian and Fichtean Leanings: Weyl on Logicism, Intuitionism, and Formalism." Philosophia Scienta 13: 85-96.

Tieszen, Richard. 2005. "Free Variation and the Intuition of Geometric Essences: Some Reflections on Phenomenology and Modern Geometry." Philosophy and Phenomenological Research 70: 153-173.

Weyl, Hermann. 1918a. "Gravitation und Elektrizität.” Sitzungsberichte der Königlich Preußischen Akademie der Wissenschaften zu Berlin: 465-480.

_. 1918b. "Reine Infinitesimalgeometrie." Mathematische Zeitschrift 2: 384-411.

_. 1918/1994. The Continuum. Mineola (NY): Dover, 1994.

-1919a. Raum-Zeit-Materie. Berlin: Springer. 133.

. 1919b. "Eine neue Erweiterung der Relativitätstheorie." Annalen der Physik 59: 101-

1923. "Gravitation and Electricity." In The Principle of Relativity, ed, Wilfrid Perrett and George Jeffrey, 200-216. London: Methuen.

-1927/1949. Philosophy of Mathematics and Natural Science. Princeton, NJ: Princeton University Press.

_. 1931. "Geometrie und Physik." Die Naturwissenschaften 19: 49-58.

. 1946/2009a. "Address at the Princeton Bicentennial Conference." In Mind and Nature, ed. Peter Pesic, 162-174. Princeton, NJ: Princeton University Press. 
1954/2009b “Insight and Reflection.” In Mind and Nature, ed. Peter Pesic, 204-221. Princeton, NJ: Princeton University Press.

1985/2012. "Axiomatic Versus Constructive Procedures in Mathematics." In Levels of Infinity, ed. Peter Pesic, 191-202. Mineola, NY: Dover.

Zahavi, Dan. 2003. Husserl's Phenomenology. Stanford: Stanford University Press. 